\title{
Research and resource needs for understanding host immune responses to SARS-CoV-2 and COVID-19 vaccines during aging
}

\author{
On 16 and 17 March 2021, the National Institute of Allergy and Infectious Diseases and the National Institute of Aging \\ convened a virtual workshop to discuss developments in SARS-CoV-2 research pertaining to immune responses in \\ older adults, COVID-19 vaccines in both aged animals and older individuals, and to gain some perspective on the \\ critical knowledge gaps that need addressing to establish scientific priorities for future research studies.
}

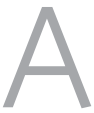

ge represents the largest known risk factor for developing severe COVID19 and is associated with higher rates of hospitalization or death following exposure to the SARS-CoV-2 virus. This is particularly evident in individuals with comorbidities, such as frailty, cardiovascular disease and diabetes. The National Institute of Allergy and Infectious Diseases (NIAID) and the National Institute of Aging (NIA) convened a virtual workshop entitled 'Immunity, Aging and COVID-19' on 16 and 17 March 2021 to discuss developments in SARS-CoV-2 research pertaining to immune responses in older adults. The goal of the meeting was to present recent findings characterizing the host immune response to SARS-CoV-2 and COVID-19 vaccines in aged animals and in older individuals, and to gain perspective on the critical knowledge gaps that could be used to establish scientific priorities for future research studies.

As noted by George Kuchel (University of Connecticut), human respiratory viruses contribute to a vast number of infections that result in a wide variety of symptoms and disease severity that may not be easily distinguished clinically. Moreover, aging influences clinical manifestations and responses to the SARS CoV-2 virus, where older adults exhibit lower fever, delirium and declines in mobility. Therefore, there is a large amount of heterogeneity among older adults infected with SARS CoV-2 virus with multimorbidity, frailty and genetic contributors (for example, the E4 allele of $A P O E$ ) serving as additional risk factors that can also lead to atypical manifestations of COVID-19. In addition, biological aging and age-related declines in immune function (immunosenescence) are thought to have a major role in the increased susceptibility to severe COVID19 outcomes observed in older individuals. Thus, improved understanding of the pathophysiology of aging and dysregulation of immune responses will help to provide novel strategies for improved therapeutic approaches and vaccine development for COVID-19.

Janet McElhaney (Health Sciences North Research Institute) mentioned important lessons learned from the longer history of influenza studies that have indicated that frailty is an important and relevant factor to be considered. When conducting COVID-19 studies, it is critical for effective vaccines to stimulate $\mathrm{CD} 8^{+} \mathrm{T}$ cell memory to improve pulmonary responses to infection while attenuating heightened systemic inflammatory responses that contribute to serious complications, as observed in influenza in older adults ${ }^{1}$. Although the robustness of $\mathrm{CD}^{+} \mathrm{T}$ cell responses to highdose seasonal influenza vaccine has been found to be linked to telomere length and correspond closely with antibody responses ${ }^{2}$ (Nan-ping Weng (NIA)), the contribution of telomere length to $\mathrm{T}$ cell memory during COVID-19 remains to be determined.

It has also become recognized that biological sex and the social construct of gender are important factors in the epidemiology and outcome of many diseases. Sabra Klein (Johns Hopkins University) noted that early observations during the pandemic reported that men were more likely to be hospitalized and die from severe COVID-19 before the availability of vaccines. Although many inflammatory markers are upregulated in men when compared to women, sex differences in severe COVID-19 become less prominent with age, while older individuals and men have greater convalescent antibody titers ${ }^{3}$.

Characterization of innate and adaptive
immune responses in aging
Janko Nikolich-Zugich (University of
Arizona) presented an overview of how
aging affects immune function at multiple
levels, including changes intrinsic to
adaptive and innate immune cell types
as well as the environment in which they

operate. Which of these aspects contributes to the enhanced susceptibility of older individuals to severe COVID-19 is not known. The Perlman laboratory (University of Iowa) has demonstrated that a deficiency in dendritic cell migration to lymph nodes and elevation of eicosanoid underlies the increased susceptibility of aged mice to the coronavirus SARS-CoV. Whether this mechanism also takes place in older individuals remains to be determined. Systems immunology approaches have proved extremely valuable in establishing immune signatures of COVID-19. Use of multimodal single-cell approaches revealed that type I interferon (IFN) and NF- $\mathrm{KB}$ signatures in several cell types, including CD56 ${ }^{\mathrm{dim}}$ natural killer cells, correlated negatively with disease severity. By contrast, plasmacytoid dendritic cell apoptosis and oxidative-stress-induced senescence correlated positively with disease severity ${ }^{4}$. Additional studies of innate immune system activation in COVID-19 infection were presented by Joseph El-Khoury (Massachusetts General Hospital). His group compared single-cell RNA-sequencing responses of peripheral blood mononuclear cells from healthy adult (25-35 years old) or older (60-80 years old) individuals to live or inactivated SARS-CoV-2 virus, viruslike particles or recombinant viral proteins. They found increased levels of chemokine and inflammatory cytokine gene expression with aging. Interestingly, recombinant spike protein alone - in the absence of infection or the ACE2 receptor - increased monocyte activation in assays performed with samples from older individuals. These studies highlighted the diverse ways by which dysregulated innate immunity contributes to COVID-19.

The role of adaptive immunity during COVID-19 infection was presented by several investigators. An adaptive immunity score that combined characteristics of $\mathrm{B}, \mathrm{CD}^{+}$and $\mathrm{CD}^{+}$cell responses was 
inversely and strongly correlated with disease severity ${ }^{5}$, suggesting that a weak adaptive immune response in older adults presented a definite risk, and that low naive $T$ cell frequencies were one feature that contributed to this risk ${ }^{5}$. Longitudinal analysis of patients convalescing from COVID-19 showed that spike-binding antibodies and memory B cells were durable for over eight months; virus-specific CD4 and CD8 T cells had a half-life of 200 days ${ }^{6}$ (Rafi Ahmed (Emory University)). Ongoing studies are addressing reactivity to SARS-CoV-2 variants with a focus on characterization of memory $\mathrm{T}$ cells and long-lived plasma cells. Patrick Wilson (University of Chicago) discussed B cell memory to SARS-CoV-2 and demonstrated that such responses were not targeted only to the spike protein but also to internal proteins. However, only antibodies to the spike protein were neutralizing and protective ${ }^{7}$. Memory responses to SARS$\mathrm{CoV}-2$ shifted to target internal proteins over time, with a shift in antigen targeting with age toward nonprotective internal antigens that were non-neutralizing and nonprotective.

The importance of autoantibodies emerged as a common theme in severe COVID-19. Ignacio Sanz (Emory University School of Medicine) presented findings in humans that both severe COVID-19 and 'long COVID' syndrome were marked by an autoimmune antibody component. Additionally, intense extrafollicular responses from several $\mathrm{CD} 27^{-} \mathrm{CD} 38^{-}$ double-negative subsets correlated with severe COVID $-19^{8}$, despite early and high levels of anti-receptor-binding-domain antibodies. For all ages, severe COVID-19 was associated with a deficiency in type I IFN responses ${ }^{9}$, and by the development of anti-IFN antibodies ${ }^{10,11}$ (Jean Laurent Casanova (Rockefeller University)). These autoantibodies, commonly seen in older individuals, suppressed the early innate response to SARS-CoV-2 infection, resulting in dysregulated inflammatory cascades. Together, these talks revealed how dysregulated inflammation both centrally (as controlled by genetic predisposition) and also as controlled locally at or near the tissue site of infection can contribute to worsening COVID-19.

\section{Comorbidities in older adults and clinical observations of post-acute sequelae}

Factors other than chronological age appear to contribute to the health status of older adults infected with SARS CoV-2. These include biological, functional, cognitive and clinical aspects that contribute to the outcome of COVID-19 with age. This session focused on the results of epidemiological and natural history studies that analyzed the association between the presence of comorbidities and adverse outcomes of COVID-19 with age. João Pedro Correa Delgado (University of Exeter) presented findings on the epidemiology of COVID-19 beyond chronological age. For individuals more than 65 years old, being male, Black and having comorbidities such as dementia, chronic obstructive pulmonary disease and type 2 diabetes significantly increased the risks of dying ${ }^{12}$, while a notable mortality risk was associated the $A P O E$ e 4/e4 genotype ${ }^{13}$. For post-acute sequelae (the long-COVID syndrome), age was a predictor, but also occurred at younger ages (40-60 years old). Sunil Ahuja (University of Texas Health Science Center San Antonio) discussed the concept of immunologic resilience and COVID-19 survival independent of age ${ }^{14}$. His group evaluated immunologic resilience using two metrics: four immune health grades (IHGs) (with IHG-I indicating preserved immunologic resilience), based on the equilibrium between CD8+ and CD4+ T cells, and by transcriptomic metrics that associated with survival in patients with COVID-19, independent of age. In persons with or without COVID-19, the best IHG (IHG-I) was more common in younger adults and women. In 526 patients with COVID-19, across age strata, presentation with IHG-I was associated with substantially lower mortality (age- and sex-adjusted mortality hazard ratio was lower by nearly $90 \%$ ) as well as hospitalization risk, shorter hospitalization, lower plasma IL-6 and rapid clearance of SARS-CoV-2 from the nasopharynx. The propensity to degrade immunologic resilience before and/or after SARS-CoV-2 is more common in men, and predisposes to severe COVID-19. Further investigation is required to assess the relationship of peripheral blood $\mathrm{T}$ cell counts and COVID-19 outcome ${ }^{15}$.

The mechanisms underlying the presentation of severe COVID-19 in both the lungs and heart remain unclear in both older and younger individuals. Data were presented on lung disease by Donna Farber (Columbia University), where longitudinal profiling of paired respiratory wash and blood samples from patients with severe COVID-19 - using highdimensional transcriptome, secretome and flow cytometry profiling - were evaluated. Patients with increased myeloid cells and lower T cell (specifically tissue-resident memory $\mathrm{T}$ cell) frequencies in the airway had worse outcomes, while immune cell frequencies in the blood did not associate with outcome. They further demonstrated ${ }^{15}$ that lung damage was associated with infiltration of aberrant monocytes from the blood into the airways through a CCL2 chemokine gradient, leading to a dysregulated inflammatory cascade. Patricia Nguyen (Stanford University) discussed the role of cardiovascular disease and atherosclerotic plaques as exacerbating inflammation that worsens COVID-19. She showed data that atherosclerotic plaques could be sources of cytokines that contributed to post-acute COVID-19 sequelae. She also identified CD4 and CD8 effector $\mathrm{T}$ cells infiltrating these plaques that may predispose to aberrant $\mathrm{T}$ cell responses ${ }^{16}$.

Together, these talks revealed how dysregulated inflammation, both centrally as controlled by genetic predisposition and controlled locally at or near the tissue site of infection, can contribute to worsening COVID-19.

\section{Preclinical animal models for evaluation of disease, vaccines and therapeutic agents}

At the beginning of the COVID-19 pandemic, new vaccines and therapeutic agents were in development and testing for safety and efficacy of these treatments required the use of animal models most appropriate for these studies. This required the identification of animal models that were not only susceptible to infection with SARS-CoV-2, but also best replicated the human disease. Using knowledge from other coronaviruses (such as SARS-CoV and MERS-CoV), it was established within months that the Syrian hamster and transgenic or transfected mice expressing the ACE2 protein were susceptible to SARS$\mathrm{CoV}-2$ infection. More important was the characterization of nonhuman primate models for preclinical testing of vaccine candidates and therapeutic agents before they could be evaluated in humans. How these models could be used to understand the effect of increasing age was also a critical question, as aging was identified as a major risk factor for severe COVID-19 disease.

Joanne Turner (Texas Biomedical Research Institute) provided an overview of available animal models for SARS-CoV-2 research, and the strengths and weaknesses of each model. She provided a review of pilot nonhuman primate studies performed at the Texas Biomedical Research Institute in early 2020, which included marmosets, rhesus macaques and baboons of two different ages ${ }^{17}$. Macaques showed mild-tomoderate symptoms before resolving disease and older macaques had reduced or delayed SARS-CoV-2 antigen-specific antibodies 
and T cell antigen-specific IL-2 production. Downstream studies from these pilot experiments are ongoing.

Luis Giavedoni (Trinity University) found that SARS-CoV-2-infected baboons were more susceptible to infection than macaques, presenting with sustained viral shedding in the gastrointestinal tract. SARS-CoV-2-infected baboons showed ageassociated changes, including an extended period with a clinical score of infection, persistent viral RNA in bronchoalveolar lavage and the nasal cavity relative to young baboons, increased pathological lesions in the lung and intestine, and elevated cytokines and chemokines in bronchoalveolar lavage. Furthermore, SARS$\mathrm{CoV}$-2-specific antibody production was blunted in older animals, whereas crossreactive antibody responses to seasonal coronaviruses were greater in older baboons. Both macaques and baboons may therefore be ideal systems to study moderate SARSCoV-2 infection, including testing of vaccine and therapeutic agents.

Kyle Rosenke (NIAID Rocky Mountain Laboratories) showed that Syrian hamsters are highly susceptible to a range of SARSCoV2 infection, which leads to moderate (10\%) weight loss and detectable virus in the lung, oral and rectal cavities for up to 10 days $^{18}$. Furthermore, the Syrian hamster model developed pulmonary tissue inflammation that resolved around 10 days after infection. Although not a lethal model, the Syrian hamster is ideally suited for screening therapeutic agents; however, the lack of immunological tools makes the Syrian hamster model less practical for understanding immune responses.

Jordi Torrelles (Texas Biomedical Research Institute) provided a summary of validation studies for the K18 hACE2transgenic mouse model. The K18 hACE2transgenic mice are highly susceptible to SARS-CoV-2 infection, exhibiting rapid weight loss and death by day 6 of infection, with detection of virus in nasal turbinates and lung that was associated with a chemokine and cytokine storm. Studies presented indicate that the K18 hACE2transgenic mice replicate the cytokine storm phenomena observed in humans, including recent reports of cytokine and chemokine production in human bronchoalveolar lavage. Lung pathology was extensive and included vasculitis and interstitial pneumonia. Of interest, K18 hACE2transgenic mice had increasing viral titers in the brain over the course of infection, detectable virus and increased presence of neutrophils and T cells within brain tissues ${ }^{19}$. This model is valuable for studying severe COVID-19, vaccine efficacy and therapeutic

Box 1 | Research gaps and opportunities to improve understanding of COVID-19 in older adults

Resource needs

- Establish repositories with clinical samples from older patients with COVID-19, including serum, plasma, cryopreserved peripheral blood mononuclear cells and banked bronchoalveolar samples.

- Establish aged animal models (for example, ACE2-transgenic mouse colonies, nonhuman primates and Syrian hamsters) for the research community.

- Develop a web portal that is interoperable with existing websites to capture SARS-CoV-2 clinical and research data on older individuals, which permits meta-analysis of reported data.

- Develop SARS-CoV-2 reagents for research (for example, antibodies, spike protein variants and antigens).

- Establish programs that promote collaboration among investigators, pharmaceutical companies and funding agencies.

Mechanistic studies of immune deficiencies in older individuals

- Cellular and molecular mechanisms of innate and adaptive immune response to SARS CoV-2 infection and vaccines in older individuals.

- Characterization of sex differences in inflammatory responses to SARS CoV-2.

- The role of epigenetic reprogramming of immune cells that promote antiviral resistance.

- Examine age-related changes in lung physiology in older adults and animal models to determine effects on viral replication and clearance (for example, reactive oxygen species, eicosanoids and so on) viral and immune physiology in lungs and upper airways in older adults and animal models.

- Characterization of duration of protective memory responses to both

agent screening, including neutralizing antibody therapies.

Bali Pulendran (Stanford University) discussed the role of immune adjuvants in promoting enhanced and durable antibody responses to subunit COVID-19 vaccines, in which the receptor-binding domain of the SARS-CoV-2 spike protein was displayed on I53-50 nanoparticles. In nonhuman primates, the use of AS03, CpG and AS37 as adjuvants yielded superior antibody titers and protection, but AS03 also induced
COVID-19 and vaccination in older adults.

- Development of animal models that capture characteristics of long-term COVID-19, including those that permit the study of aging in a clinically relevant context of heterogeneity (for example, genetic background, comorbidity and cognitive decline).

- Immune and inflammatory characterization of long-term COVID-19 complications.

- Long-term studies for assessing persistence of COVID-19 symptoms, reservoirs for virus persistence and the durability of immunity to evolving variants.

Clinical studies

- Role of previous coronavirus infections in ability of older individuals to mount a protective response to SARS-CoV-2 and vaccines.

- Association of vaccine efficacy and durability of responses in older adults and individuals with frailty and/or other comorbidities.

- Evaluate differences in immunological consequences of individuals living in long-term care facilities compared to those living at home.

- Determine the efficacy of combination therapies, including targeting the innate immune system to improve host immune function in older adults.

- Evaluate the role of interventions targeting various hallmarks of aging for their ability to improve outcomes of severe COVID-19 among vulnerable older adults.

- Clinical studies modulating inflammatory mediators during SARS CoV-2 infection in older adults to improve outcomes.

superior cross-neutralization of variant viruses compared to $\mathrm{CpG}$ alum and $\mathrm{AS} 37^{20}$. Two phase I/II clinical trials have been initiated (NCT04742738 and NCT04750343) on the basis of these results.

The workshop session identified a series of roadblocks or gaps in knowledge from animal model development for SARS-CoV-2 infection, or gaps in knowledge in humans where animal models may have significant value. An important finding is that all of the nonhuman primate models tested were 
quite resistant to SARS-CoV-2 infection and are therefore limited to the study of mildto-moderate human disease, or vaccine and therapeutic agent screening. Furthermore, all nonhuman primate work has to date been short-term and there is a need for long-term studies (Box 1).

\section{COVID vaccine effectiveness in older adults}

Presentations in this session discussed promising data indicating that effectiveness of the current COVID-19 vaccines is, in general, comparable in older and younger adults, and that even a single dose provides some level of protection in older adults. However, the older adult populations studied to date have been relatively healthy and may not represent vaccine efficacy for frail older adult populations, those with additional comorbidities or those residing in nursing homes.

Joan Mannick (Life Biosciences) presented data analyzing the effectiveness of the oral mTOR inhibitor RTB101 at enhancing antiviral immune responses in older adults. Older adults are susceptible to viral respiratory tract infections owing to an attenuated type I IFN immune response to viruses ${ }^{21}$, which has been shown to be particularly important for fighting SARSCoV-2 infections. Previous phase IIb and phase III trials conducted in over 1,500 adults aged 65 years or older demonstrated that RTB101 was well-tolerated and consistently upregulated IFN-induced antiviral gene expression in older adults ${ }^{21}$. Analysis of serum samples from the phase III trial revealed that RTB101 treatment increased IFN $\alpha$ levels only in older adults who had a respiratory tract infection. Ex vivo studies of peripheral blood revealed that the primary cell type that produced IFN $\alpha$ in response to RTB101 treatment was plasmacytoid dendritic cells in combination with the viral mimetic R848. On the basis of these encouraging results, a clinical trial was undertaken to assess whether prophylaxis with RTB-101-210 decreased the severity of COVID-19 in residents of nursing homes experiencing a COVID-19 outbreak ${ }^{22}$.

John Beigel (NIAID) subsequently discussed age-related responses to the Moderna COVID-19 mRNA vaccine. In phase I trials, antibody titers to spike protein and neutralizing antibodies were comparable in people aged 55 years or younger and people aged 56-70 years. In all age groups, $\mathrm{T}$ helper 1 cell - and not T helper 2 cell responses were elicited. There was a lower incidence of local and systemic adverse effects following vaccination in people aged 55 years or older. In the phase III trial, vaccine efficacy was $95.6 \%$ (95\% confidence interval 90.6-97.9) in people aged 18 to less than 65 years and $86.4 \%$ (61.4-95.2) in people aged 65 years or older ${ }^{23}$. Thus, in older populations, the efficacy of the mRNA-1273 vaccine appears to be similar in all age groups; however, the smaller numbers of cases that occurred in older adults limits the ability to determine whether a difference exists in this subgroup.

Keipp Talbot (Vanderbilt University) provided additional data on the effectiveness of the Pfizer/BioNTech BNT162b2 and the Janssen Ad26.COV2.S vaccines in older adults. The effectiveness of the BNT162b2 vaccine was similar in younger and older adults. However, the older adult populations in the studies were relatively healthy: $36 \%$ of cohort of participants aged 65-74 years, and $47 \%$ of the cohort of participants aged 75 years or older, had comorbidities.

The effectiveness of the Janssen Ad26. CoV2.S vaccine for preventing symptomatic disease was also similar in younger and older cohorts. However, in the cohort aged 60 years or older, there was some evidence (although the confidence intervals were large) that vaccine effectiveness for preventing severe disease was lower in individuals who had comorbidities. Specifically, vaccine effectiveness was $100 \%$ (42.5-100.0) in individuals aged 60 years or older without comorbidities, but only $57.1 \%(-88.0-92.8)$ in individuals with comorbidities. Talbot presented some real-world data for various vaccines in older adult populations. In long-term care facilities in Denmark, the vaccine efficacy was higher among the staff than among the residents, suggesting decreased efficacy in more frail elderly populations. A limitation of vaccine trials in older adults is that they often exclude individuals living in nursing homes, patients with high expected mortality, patients taking any form of immune suppression, patients with cancer, patients with dementia and individuals with impaired mobility who have difficulty traveling to study sites. Talbot recommended that future trials be conducted in the intended population and include measures of frailty and immunosenescence in the study participants.

Finally, Teresa Lambe (University of Oxford) presented data for the AZD1222 vaccine and discussed promising data on the real-world vaccine effectiveness after a single dose in older adults in the UK. The AZD1222 vaccine has fewer side effects in people 70 years or older than in people aged 18-55 years ${ }^{24}$. Lambe summarized data from the EAVE II, AVON CAP and Public Health England studies that included wide age ranges as well as frail older adults. Both the BNT162b2 and AZD1222 vaccines were comparable in their efficacy, with a slight advantage of AZD1222 in individuals over the age of 80. These studies also examined vaccine efficacy after a single dose, finding it to be in the range of $80 \%$ in most cases.

\section{Concluding remarks}

Several research gaps and challenges were discussed at the workshop (Box 1). The COVID-19 pandemic has brought the concepts of frailty, multimorbidity and geroscience to the fore and highlighted various research gaps that need to be addressed for COVID-19. The inclusion of older individuals with frailty and multiple coexisting chronic conditions in clinical studies for therapeutic treatments for COVID-19 or vaccine studies should be implemented. Although older adults were more susceptible to delirium and coma upon ventilation, the COVID-D study ${ }^{25}$ demonstrated that decreasing modifiable risk factors provides opportunities for reducing brain dysfunction in these patients (Wes Ely (Vanderbilt University)). The role of biological aging as a driver of this type of vulnerability has opened opportunities for the testing of geroscience-guided interventions as a means of overcoming aging-related declines in immune responses to COVID-19 as well as other pathogens, thereby improving physiologic resilience and clinical outcomes in this population.

The development of new strategies for broader disease prevention and control in vulnerable older adults will require not only dedicated resources but also will necessitate new approaches enabled by multidisciplinary efforts spanning traditional disciplines and siloes.

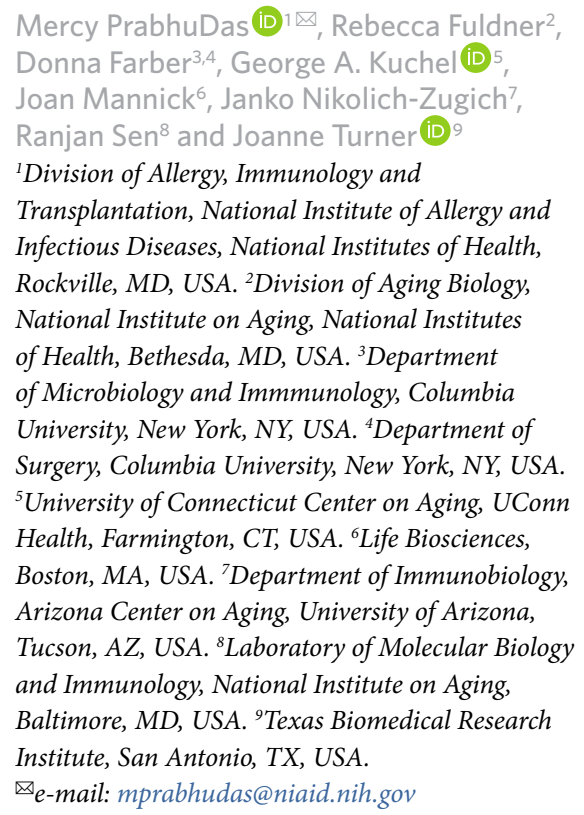


Published online: 22 December 2021

https://doi.org/10.1038/s43587-021-00156-x

References

1. McElhaney, J. E. et al. Immun. Ageing 17, 10 (2020).

2. Najarro, K. et al. J. Infect. Dis. 212, 1261-1269 (2015).

3. Klein, S. L. et al. J. Clin. Invest. 130, 6141-6150 (2020)

4. Liu, C. et al. Cell 184, 1836-1857 (2021).

5. Rydyznski Moderbacher, C. et al. Cell 183, 996-1012 (2020).

6. Cohen, K. W. et al. Cell Rep. Med. 2, 100354 (2021).

7. Dugan, H. L. et al. Immunity 54, 1290-1303 (2021).

8. Woodruff, M. C. et al. Nat. Immunol. 21, 1506-1516 (2020).

9. Asano, T. et al. Sci. Immunol. 6, eabl4348 (2021).

10. Bastard, P. et al. J. Exp. Med. 218, e20202486 (2021).

11. Bastard, P. et al. J. Exp. Med. 218, e20210554 (2021).

12. Kuo, C. L. et al. J. Gerontol. A Biol. Sci. Med. Sci. 76, e133-e141 (2021)

13. Kuo, C.-L. et al. J. Gerontol. Series A 75, 2231-2232 (2020).

14. Lee, G. C. et al. J. Allergy Clin. Immunol. 148, 1176-1191 (2021).

15. Szabo, P. A. et al. Immunity 54, 797-814.e6 (2021).

16. Zhu, H. et al. Curr. Cardiol. Rep. 22, 32 (2020).

17. Singh, D. K. et al. Nat. Microbiol. 6, 73-86 (2021).
18. Rosenke, K. et al. Emerg. Microbes Infect. 9, 2673-2684 (2020)

19. Oladunni, F. S. et al. Nat. Commun. 11, 6122 (2020). 20. Arunachalam, P. S. et al. Nature 594, 253-258 (2021). 21. Mannick, J. B. et al. Sci. Transl. Med. 10, eaaq1564 (2018). 22. Cox, L. S. et al. Lancet Healthy Longev. 1, e55-e57 (2020). 23. Anderson, E. J. et al. N. Engl. J. Med. 383, 2427-2438 (2020). 4. Ramasamy, M. N. et al. Lancet 396, 1979-1993 (2020). 25. Pun, B. T. et al. Lancet Respir. Med. 9, 239-250 (2021).

Acknowledgements

We thank all workshop participants for their expertise and insights on the topic of aging, immune responses and COVID-19. Workshop participants included J. McElhaney, G.A.K., N.-p. Weng, S. Klein, J.N.-Z., S. Perlman, J. El-Khoury, R. Ahmed, P. Wilson, I. Sanz, J. L. Casanova, J. P. Correa Delgado, S. Ahuja, D.F., P. Nguyen, J.T., L. Giavedoni, K. Rosenke, J. Torrelles, B. Pulendran, J.M., J. Beigel, K. Talbot, T. Lambe and W. Ely. We thank the NIAID audio-visual team for their expert assistance throughout the workshop. The organization of this meeting was supported by NIAID and NIA of the
US National Institutes of Health (NIH). We thank B. Shea Sullivan (NIH Library) for manuscript editing assistance. This report is the summary of the collective views from the meeting participants and does not necessarily reflect the views of NIAID or NIA.

Author contributions

All authors drafted the manuscript. M.P., R.F. and R.S. substantively revised the manuscript, and all authors have approved the revised, submitted manuscript.

\section{Competing interests}

G.A.K. has served as consultant to Janssen Pharma, Spring Discovery and ResTORBio. J.M. owns equity in Adicet, which is the owner of RTB101; J.N.Z. is co-chair of the Scientific Advisory Board and his research is partially supported by Young Blood Institute, Inc. These companies had no influence or impact in any manner on the content or design of this manuscript. All remaining authors declare no competing interests. 